\title{
Research on Influence Factors of the Elderly's Intention to Use Mobile APPs
}

\author{
Kaixuan Wang \\ School of Economics and Management, Beijing Jiaotong University, Beijing 100044, China \\ 14120578@bjtu.edu.cn
}

\begin{abstract}
This paper researches into the elderly's intention to use mobile APPs and adds the theory of perceived playfulness and theory of perceived risk to UTAUT to create a theoretical model. Through questionnaire survey and using SPSS and AMOS for statistical analysis and structural equation model analysis, this paper examines the hypothese of the theoretical model and utilizes the data analysis results to provide suggestions on mobile APPs specifically designed for the elderly.
\end{abstract}

Keywords: the Elderly, Mobile APPs, UTAUT, Intention to Use

\section{Introduction}

The fast development of mobile internet has resulted in rapid rise of the mobile APPs industry. Faced with such a strong growth and given the seriously aging Chinese society, the influence factors of the elderly's intention to use mobile APPs represent an issue meriting research.

This paper mainly looks at the influence factors of the elderly's intention to use mobile APPs, adds the theories of perceived playfulness, perceived risk and perceived cost on the basis of UTAUT model to create a theoretical model and identify the factors affecting the elderly's intention to use mobile APPs through questionnaire survey process in order to provide suggestions and a point of reference for mobile APPs specifically designed for the elderly.

\section{Model and hypothesis}

\subsection{Modeling}

United Theory of Acceptance and Use of Technology (UTAUT) is a theoretical model proposed by Venkatesh by integrating TTF, TPB, DOI and STC theories on the basis of the technology acceptance model proposed by Davis. The UTAUT model consists of four essential variables: performance expectancy, effort expectancy, social influence and facilitating conditions.

Numerous studies suggest that the UTAUT model has a strong ability to interpret users' intention to use information technology, therefore this paper will look into the elderly's intention to use mobile APPs based on the UTAUT model.

This paper will also add the perceived playfulness, perceived risk and perceived cost to the UTAUT model to create a model for the elderly's intention to use mobile APPs. The research model design in this paper is as follows: 


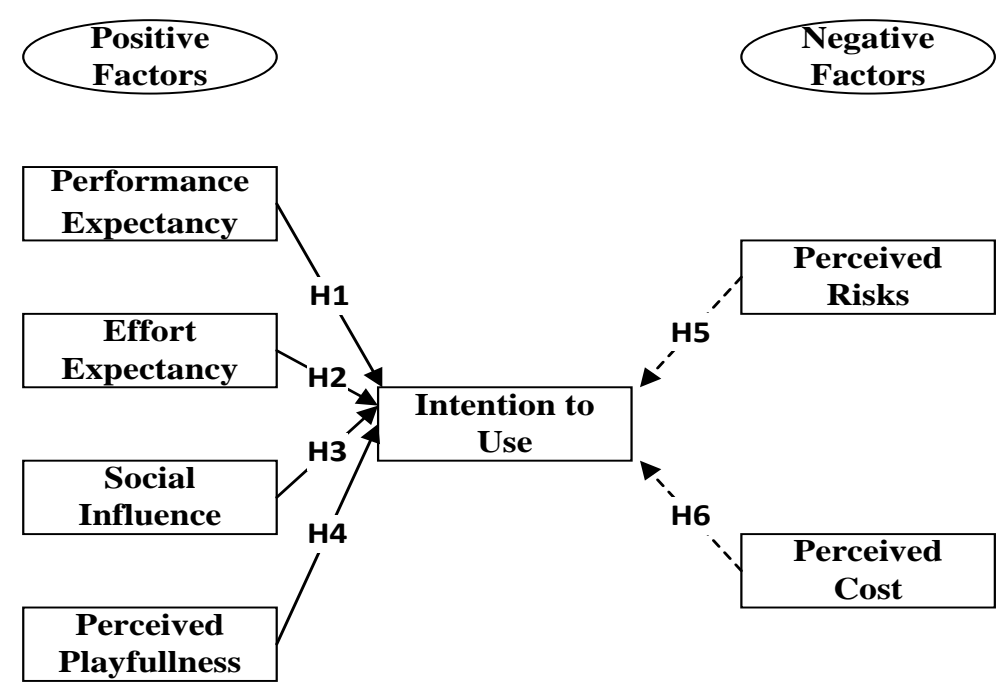

Fig. 1 The model for influence factors of the elderly's intention to use mobile APPs

\subsection{Research hypotheses}

\subsubsection{Based on UTAUT model}

In the UTAUT model, the three factors of performance expectancy, effort expectancy and social influence all positively influence the user's intention to use. In this research, the aforesaid four measurement variables are defined as follows:

Intention to use means the possibility that the elderly believe they will use mobile APPs in the future; Performance expectancy refers to the extent to which use of mobile APPs brings convenience and help to the lives of the elderly; Effort expectancy indicates the extent of difficulty or ease with which the elderly think they use mobile APPs; Social influence means the extent of support that the elderly consider their families and friends have for mobile APPs.

Hence, this paper proposes the following hypotheses:

H1: The elderly's performance expectancy for mobile APPs will positively influence their intention to use mobile APPs.

H2: The elderly's effort expectancy for mobile APPs will positively influence their intention to use mobile APPs.

H3: Social influence will positively influence the elderly's intention to use mobile APPs.

\subsubsection{Based on Perceived Playfulness}

In this paper, perceived playfulness refers to the elderly's experience in the process of mobile APPs use: if the elderly feel that the use of mobile APPs is a very enjoyable operation and that a sense of freshness will be brought to them in this process, they will be more inclined to use. Therefore, the following hypothesis is proposed:

H4: The elderly's perceived playfulness for mobile APPs will positively influence their intention to use mobile APPs.

\subsubsection{Based on Perceived Risks}

Perceived risks refer to the losses that the elderly subjectively expect to suffer while using mobile APPs. When using mobile APPs, the elderly would be concerned about the use of data and possible loss of smartphone, thus incurring financial risk; due to complicated interface and difficulty to operate, they would feel frustrated and incur mental risk in the use process; use of mobile APPs will consume time and energy and might result in leakage of personal information, thus incurring the risk of time and security. Once realizing that use of mobile APPs will generate a certain risk described above, the elderly might give up the idea of tryout and use. Therefore, the following 
hypothesis is proposed:

H5: The elderly's perceived risks for mobile APPs will negatively influence their intention to use mobile APPs.

\subsubsection{Based on Perceived Cost}

The cost in this paper mainly consists of time cost and financial cost. Time cost refers to the time that the elderly spend on using mobile APPs; financial cost refers to the data fee incurred by the elderly while using mobile APPs and the cost of use of paid APPs as well as the cost of purchase of smartphone. If the elderly feel the cost of use of mobile APPs exceeds that expectation, they would not attempt to use. Therefore, the following hypothesis is proposed:

H6: The elderly's perceived cost for mobile APPs will negatively influence their intention to use mobile APPs.

\section{Empirical analysis}

\subsection{Questionnaire design and sample statistics}

This research employs questionnaire survey to test the model of influence factors of the elderly's intention to use mobile APPs as proposed above. The model in Figure 2 consists of 7 potential variables, i.e., performance expectancy (PE), effort expectancy (EE), social influence (SI), perceived playfulness (PP), perceived risks (PR), perceived cost (PC) and intention to use (IU). The final questionnaire contains 25 measurement questions, each employing Likert five-point scale for measurement from highly disagree (1 point) to highly agree (5 points).

To ensure the reasonableness of the questionnaire structure and scale design, before formal survey, a pre-survey was conducted for the initially designed questionnaire. This paper mainly employs offline survey process in which 400 copies of questionnaire were distributed offline and 373 copies recovered, of which 306 are valid. The statistical description of the questionnaire is shown in the following table:

Table 1 Sample statistical description

\begin{tabular}{ccll}
\hline Basic information & Option & Frequency & Percentage (\%) \\
\hline \multirow{2}{*}{ Sex } & Male & 149 & 48.7 \\
& Female & 157 & 51.3 \\
\hline \multirow{2}{*}{ Age } & $60-70$ & 178 & 58.2 \\
& $70-80$ & 93 & 30.4 \\
& above 80 & 35 & 11.4 \\
\hline \multirow{3}{*}{ Education } & Elementary School & 39 & 12.7 \\
& Junior School & 104 & 34.0 \\
& High School & 92 & 30.1 \\
& College and Above & 71 & 23.2 \\
\hline \multirow{2}{*}{ Income } & Less than 1000 & 18 & 5.8 \\
& 1000-3000 & 181 & 59.2 \\
& More than 3000 & 107 & 35.0 \\
\hline
\end{tabular}

\subsection{Data analysis}

\subsubsection{Questionnaire reliability and validity analysis}

Reliability refers to the consistency and stability of measurement results obtained based on the scale. The higher the scale reliability, the more stable the scale is. In the Likert scale, the commonly used reliability test method is Cronbach's $\alpha$ coefficient. It is generally believed that over 0.7 of $\alpha$ coefficient means the reliability is acceptable, over 0.8 means the reliability is relatively high and 
over 0.9 means the reliability is very ideal. The empirical data was tested using SPSS18.0, resulting in $\alpha$ coefficient of each potential variable as shown in Table 2 . It can be seen from Table 2 that $\alpha$ coefficient of each variable is above 0.8, meaning the reliability of each of 7 variables is rather ideal.

Table 2 Cronbach's $\alpha$

\begin{tabular}{|c|c|c|}
\hline Variables & Number of questions & Cronbach's $\alpha$ \\
\hline $\mathrm{PE}$ & 4 & 0.887 \\
\hline EE & 3 & 0.882 \\
\hline SI & 3 & 0.906 \\
\hline PP & 5 & 0.835 \\
\hline PR & 4 & 0.829 \\
\hline PC & 3 & 0.884 \\
\hline BI & 3 & 0.921 \\
\hline
\end{tabular}

To further test the reliability and validity of the scale, CFA analysis was conducted for the model using AMOS17.0, indicating that the standard load value of measurement item of each potential variable is above 0.5 and significant at the level of 0.001 . Table 3 gives the average variance extracted (AVE) value and the composite reliability (CR) value of each potential variable. The AVE value of each variable is above 0.5 , indicating that the measurement model has a good reliability and convergent validity. Meanwhile, the CR value of each variable is above 0.8 , indicating each variable has a very good internal consistency.

Table 3 AVE and CR of each variable

\begin{tabular}{lcc}
\hline Variables & AVE & CR \\
\hline PE & 0.5420 & 0.8823 \\
EE & 0.7231 & 0.8423 \\
SI & 0.5875 & 0.8537 \\
PP & 0.5736 & 0.9024 \\
PR & 0.7437 & 0.8747 \\
PC & 0.6728 & 0.9126 \\
BI & 0.7241 & 0.9127 \\
\hline
\end{tabular}

\subsubsection{Structural equation model analysis}

This research employs AMOS17.0 for SEM analysis and validation and to test the model hypotheses. The goodness of fit index and corresponding acceptable suggested value are shown in Table 4. 
Table 4 Goodness of Fit Analysis and Measure Index

\begin{tabular}{lllr}
\hline GFI & \multicolumn{1}{c}{ Ideal standard value } & Acceptable standard value & Results \\
\hline$\chi^{2}$ & N/A & N/A & 2654.274 \\
df & N/A & N/A & 999 \\
$\chi^{2} /$ df & $\leq 2.00$ & $\leq 3.00$ & 2.657 \\
RMSEA & $\leq 0.05$ & $\leq 0.08$ & 0.064 \\
AGFI & $\geq 0.80$ & $\geq 0.70$ & 0.718 \\
NFI & $\geq 0.90$ & $\geq 0.80$ & 0.851 \\
RFI & $\geq 0.90$ & $\geq 0.80$ & 0.839 \\
CFI & $\geq 0.90$ & $\geq 0.80$ & 0.901 \\
IFI & $\geq 0.90$ & $\geq 0.80$ & 0.901 \\
PGFI & $\geq 0.5$ & & 0.664 \\
PNFI & $\geq 0.5$ & & 0.786 \\
PCFI & $\geq 0.5$ & & 0.833 \\
\hline
\end{tabular}

All important fit indices are within the range of acceptable suggested values, therefore the theoretical model and the empirical data have relatively high degree of fitting. Through path relation analysis of the model and validation of the hypothetical variable relation based on the path coefficient result, the hypothetical test results are shown in Table 5. It can be seen from the information contained in Table 5 that all of the six hypotheses of the theoretical model passed the $\mathrm{T}$ test and are fully supported.

Table 5 Hypotheses Test Results of the Model

\begin{tabular}{lccc}
\hline Hypotheses & Paths & Estimated Value & Results \\
\hline H1 & $\mathrm{BI} \leftarrow \mathrm{PE}$ & 0.828 & Support \\
H2 & $\mathrm{BI} \leftarrow \mathrm{EE}$ & 0.923 & Support \\
H3 & $\mathrm{BI} \leftarrow \mathrm{SI}$ & 0.809 & Support \\
H4 & $\mathrm{BI} \leftarrow \mathrm{PP}$ & 0.831 & Support \\
H5 & $\mathrm{BI} \leftarrow \mathrm{PR}$ & -0.807 & Support \\
H6 & $\mathrm{BI} \leftarrow \mathrm{PC}$ & -0.761 & Support \\
\hline
\end{tabular}

\subsubsection{Result analysis and practical significance}

All of the six hypotheses proposed by this research have been validated. Of them, the effort expectancy is the most important factor influencing the elderly's intention to use mobile APPs. This indicates that the elderly care about the degree of operational ease or difficulty while using mobile APPs and that the complicated operation and lack of targeted design will reduce the elderly's intention to use mobile APPs. As age grows, the elderly's vision and physical flexibility will remarkably decrease, therefore APP interfaces need to have clearly defined boundaries and a high contrast when developing mobile APPs for the elderly in order for the elderly to accurately distinguish the background and objectives of the mobile phone interfaces and avoid various operational errors.

The positive influence of performance expectancy is also remarkable, indicating that the elderly are remarkably concerned about whether mobile APPs will bring them convenience and help in daily life. The vast varieties of mobile APPs basically cover every aspect of daily life and can greatly facilitate the elderly's daily life. Therefore, to increase the elderly's performance expectancy for use of mobile APPs, more functions should be added to mobile phone. For example, as the elderly pay much attention to physical health, it is advisable to add health information and the physical indicator measurement capability to mobile phone. 
Social influence remarkably affects the elderly's intention to use mobile APPs, indicating that the elderly's decision as to whether or not to use mobile APPs is largely affected by their families and friends. For this reason, word-of-mouth marketing may be utilized to increase the elderly's use of mobile APPs. To crease a benign word-of-mouth marketing chain, the most essential step is to ensure product quality, which requires mobile APPs providers to continuously improve APP design based on the feedback from the elderly in the use process to make them more suitable for the elderly, thus resulting in good word of mouth among them.

Currently, there are various mobile APPs on the market, which can substantially meet people's needs for information, online social activities and entertainment. The elderly's perceived playfulness while using mobile APPs has greatly increased, so the perceived playfulness has a strong influence on their intention to use mobile APPs. New experience will attract more users, meaning that the content and functionality need to be made more playful when designing mobile APPs for the elderly and more interesting elements need to be incorporated when adding new features from the perspective of the elderly in order to increase the elderly's intention to use mobile APPs.

The perceived risks and perceived cost have negative impact on the elderly's intention to use mobile APPs, indicating that the elderly are rather concerned about the negative factors brought by use of mobile APPs. These negative factors mainly come from privacy, economic and time considerations, which require the mobile APPs designers to focus on the security of APP, not to disclose the user's personal information or arbitrarily charge users while at the same time monitoring the time of use and reminding the elderly in time to avoid their indulgence in mobile APPs.

\section{Conclusion}

The model of influence factors of the elderly's intention to use model phone APP based on UTAUT model and theories of perceived playfulness, perceived risks and perceived cost created in this research effectively analyzes the influence factors of the elderly's intention to use mobile APPs, looks at the elderly's acceptance of new things from the perspective of technology acceptance and effectively predicts the elderly's intention to adopt and use mobile APPs, thus informing the design of mobile APPs developed for the elderly. Meanwhile, this research has certain limitations. In addition to the six factors considered in this paper, there are actually many other factors affecting the elderly's intention to use mobile APPs, such as family factors and physical state, which are highly likely to remarkably affect the elderly's intention to use mobile APPs. Moreover, the dimensional decomposition of the acceptance factor, risk factor and content factor already considered in this research also warrant further exploration and digging. Furthermore, as the samples involved in the questionnaire survey used in this paper are mainly in Beijing, it is advisable to consider expanding the quantity and distribution of the samples in the future to obtain more universal results in order to make this research more universally applicable.

\section{References}

[1]Boulton-Lewis G M, Buys L, Lovie-Kitchin. Ageing, learning and computer technology in Australia [J]. 33(03):253-270.

[2]White J, Weatherall A. A grounded theory analysis of older adults and information technology [J]. 26(04):371-386.

[3]Selwyn N, Gorard S, Furlong J. Older adults' use of information and communications technology in everyday life [J]. 23(05):561-582. 
[4]Leist AK. Social media use of older adults: a mini-review [J]. Gerontology,2013;59(4):378-84 . [5]Chin-Lung Hsu, Hsi-Peng Lu, Huei-Hsia Hsu. Omega: The international journal of management science [J]. 715-726.

[6]Lu YH, Xu HM. An Empirical Research on the User' s Behaviors of Instant Message Services[J].Chinese Journal of Management, 2006,(5):614-621.

[7]Yang SD, Hu C. Empirical study of users' acceptance model on mobile instant messaging services [J]. Journal of Beijing University of Posts and Telecommunications,2009,(1):33-40.doi:10.3969/j.issn.1008-7729.2009.01.007.

[8]Chen P. A Study on Weinxin User Adoption Based on Mobile Internet[D].Huazhong University of Science and Technology,2012:1-54.

[9]VENKATES V, DAVIS F D. A Theoretical Extension of the Technolo-gy Acceptance Model: for Longitudinal Field Studies [J]. 46(02):186-201.

[10]DAVIS E D, BAGOZZI R P, WARSHAW P R. User Acceptance of Computer Technology: A Comparison of Two Theoretical Models [J]. (35):982-1003.

[11]Hennington A, Janz B D. Information Systems and Healthcare XVI: Physician Adoption of Electronic Medical Records: Applying the UTAUT Model in a Healthcare Context [J]. Communications of the Association for Information Systems, 2007, 19(1): 60-80.

[12]Garfield, M. J. Acceptance of Ubiquitous Computing [J]. Information Systems Management, 2005,22(4):24-31.

[13]Zhang MR,Zhang J,Yang YN,et al. Empirical Research on User Behavior Model of Mobile Library Based on UTAUT [J]. Library Forum,2017,(6):70-77.

[14]Tan CH,Zhang J,Zeng YT. Influence Factors of Consumers' Online Shopping Based on UTAUT [J]. Modernization of Management,2014,(3):28-30. 